\section{Aesthetic empowerment through music}

Elvira Brattico

Center for Music in the Brain (MIB), Aarhus University, Denmark

Aarhus University \& The Royal Academy of Music Aarhus/Aalborg, Aarhus, Denmark
Musicae Scientiae 2019, Vol. 23(3) 285-303

(C) The Author(s) 2019

Article reuse guidelines: sagepub.com/journals-permissions DOI: I0.II77/I0298649/9850606 journals.sagepub.com/home/msx

@SAGE

\title{
Ulrika Varankaitè
}

Kaunas University of Technology, Lithuania

Vilnius Gediminas Technical University, Lithuania

\begin{abstract}
This article describes how aesthetic enjoyment accompanying musical activities can empower individuals in health and disease. First, we explain the biological determinants of music enjoyment and how they can be studied. In doing so, we distinguish between core sensory pleasure and conscious liking, and we illustrate their respective contributions to aesthetic appreciation and expressive interaction with music. Second, we review findings illustrating the long-term impact of listening to favourite music on the brains of healthy musicians as well as on the brains and behaviour of individuals with pathological symptoms. Based on these findings, we propose a set of putative neural mechanisms underlying aesthetic empowerment through music. We also emphasize the importance of considering individual differences in sensitivity to aesthetic experiences of music, as evidenced, for instance, by neuroimaging and imaging genetics studies, drawing the connections between dopamine neurotransmission and dopaminergic genes with the reactivity of music-brain functions.
\end{abstract}

\section{Keywords}

Aesthetics, neuroaesthetics, liking, pleasure, enjoyment, clinical applications

\section{Aesthetic responses to music as a basic phenomenon in life}

Music accompanies human beings throughout their lifespan as a (mainly) auditory channel for expression and communication. Infants enjoy music and are calmed down by tunes (Trehub, 2003). Individuals of all ages, with a peak in adolescence, use music in everyday life to expose themselves to a perceptual, cognitive, emotional and evaluative experience - even a motoric experience, when the act of playing an instrument is involved. This individual experience of music can be defined as aesthetic (Brattico, Bogert, \& Jacobsen, 2013), since it incorporates

\section{Corresponding author:}

Elvira Brattico, Center for Music in the Brain (MIB), Department of Clinical Medicine, Aarhus University, Nørrebrogade 44, Bld. 10 G, fl.5, Aarhus C, 8000, Denmark.

Email: elvira.brattico@clin.au.dk 
specific features that makes it similar to other experiences of other art forms. While the act of listening to music is often incidental (e.g., while roaming in a shopping mall) or functional (sought for other secondary goals, such as seducing a partner or maintaining the right pace and energy while jogging), it can also be pursued for its own sake, namely for purely aesthetic reasons of reaching an affective and evaluative response. When playing an instrument, attentional focus and the explicit or implicit intention to reach specific aesthetic goals occur together; although other types of playing do exist, such as the mechanical playing of an instrument to improve technique. Nevertheless, in some cases even incidental listening, namely not focused on attributing value and intentions to sounds, can lead to an aesthetic response of pleasure (Liu et al., 2017). According to Brattico and Pearce (2013, p. 54), an aesthetic experience is possible "without making an aesthetic decision or judgment about the stimulus causing that experience”. Indeed, when we listen to music in a restaurant, we might be immersed in its soothing atmosphere or struck by the sheer beauty or virtuosity of the jazz master's performance. However, several authors consider that the act of attributing value, meaning and intention to an artwork is intrinsic to the aesthetic experience, whether the person having the experience is an agent or a recipient (in relation to figurative arts: Snapper, Oranç, Hawley-Dolan, Nissel, \& Winner, 2015; Bloom, 1996; Preissler \& Bloom, 2008; in relation to music: Brattico \& Pearce, 2013; Brattico, 2015; Reybrouck \& Brattico, 2015; Brattico et al., 2013; Nieminen, Istók, Brattico, Tervaniemi, \& Huotilainen, 2011; Brattico, in press).

According to the neural-chronometry framework for music proposed by Brattico and colleagues (2013), this musical aesthetic experience results in three outcomes: aesthetic emotions (e.g., enjoyment, chills, nostalgia, awe, being moved; but see Koelsch (2014) and Juslin (2013a) for divergent views on aesthetic emotions); aesthetic judgements (i.e., the conscious evaluation of the beauty of a piece of music based on its formal properties); and preference (the conscious liking or disliking of a musical piece) (Brattico et al., 2016; Liu, Abu-Jamous, Brattico, \& Nandi, 2017; Liu et al., 2017). Several scholars distinguish aesthetic emotions from utilitarian, basic everyday emotions (Brattico \& Pearce, 2013; Koelsch, 2014; Schindler et al., 2017; Scherer \& Zentner, 2008). Awe, nostalgia and enjoyment (or pleasure) have been studied in more detail than other aesthetic emotions. Aesthetic awe, which, as an emotion, plays an important part in social regulation (Habibi \& Damasio, 2014), is rare but at the same time a crucial feature that characterizes a peak aesthetic experience that can be induced by especially beautiful music. Nostalgia, the sweet sorrow associated with remembering past events, is consistently triggered by musical activities, more so than other negative emotions (Taruffi \& Koelsch, 2014). The aesthetic emotion of enjoyment or pleasure has received much attention from music researchers and has been studied in relation to the perceiver's internal context, stimulus features and other emotions, but to a much lesser extent in relation to other aesthetic responses, in contrast with studies in the domain of visual arts (Tiihonen, Brattico, Maksimainen, Wikgren, \& Saarikallio, 2017). Moreover, musical pleasure has mostly been studied by focusing on the experience of chills, a physiological reaction that can be referred to as goose bumps or shivers down the spine (Panksepp \& Bernatzky, 2002; Grewe, Nagel, Kopiez, \& Altenmüller, 2005; Salimpoor, Benovoy, Longo, Cooperstock, \& Zatorre, 2009; Sumpf, Jentschke, \& Koelsch, 2015; Blood \& Zatorre, 2001). Chills have also been described as "subtle nervous tremors caused by intense emotion" (Grewe, Nagel, Kopiez, \& Altenmüller, 2007, p. 297) or even as "skin orgasms" (Harrison \& Loui, 2014). Although not every person experiences these kinds of response to music (Goldstein, 1980; Sloboda, 1991; Grewe et al., 2007), they have attracted the attention of scientists for their within-subject reliability and for the possibility they allow of translating subjective emotional experiences of pleasure into physiological measures of changes in the autonomic nervous system that can be measured objectively. Results consistently indicate that 
chills are related to the experience of increased subjective emotion and pleasure (Blood \& Zatorre, 2001), as well as to enhanced physiological arousal (Grewe et al., 2005; Salimpoor et al., 2009; Rickard, 2004; Mori \& Iwanaga, 2017).

Related to this, several researchers (Brattico \& Pearce, 2013; Brattico, 2015; Brattico et al., 2013; Stark, Vuust, \& Kringelbach, 2018) proposed that two types of pleasure can be identified. Immediate sensory pleasure or displeasure, or core "liking", refers to the bottom-up, automatic reaction to physical, acoustic features of the perceived music. The immediate reaction to a rough, inharmonic sound pattern - described by Terhardt (1974) and Plomp (1976) as sensory dissonance - refers to this kind of pleasure. In turn, conscious pleasure or enjoyment is a top-down aesthetic response, which involves reflecting on the music as it is being perceived, possibly accompanied by the wish to repeat it ("wanting”) (Kringelbach \& Berridge, 2017). Hence, conscious pleasure is derived not only from the sensory features of the stimulus but also from the listener's cultural and personal background (mood, personality, attitudes towards musical genres, socio-cultural background, past experiences) and from other concurrent emotional responses induced by the perceived music. For instance, the positive emotion derived from pondering the stylistic congruence of chords against their context, and previous knowledge of that musical style (sensory dissonance; cf. Terhardt, 1974; Plomp, 1976), illustrates this kind of enjoyment. Such a dual view of musical pleasure calls for a biologically comparative approach, such as the one described by Fitch (2015), namely for investigating musical pleasure in a wide range of non-human animal species. It is deemed possible that other species with similar to human sensory mechanisms could derive at least sensory pleasure from selfmade music-like sounds.

Juslin and Västfjäll's (2008) framework also implicitly distinguishes two kinds of mechanism whereby listeners derive pleasure and other emotions from music. The first involves early affective responses that they describe as "brainstem reflexes" (Juslin \& Västfjäll, 2008, p. 588), producing fast, automatic reactions to aversive sound features. The second involves slower mechanisms underlying cognitive types of pleasure. These include evaluative conditioning (repeated pairing of an emotional state with a specific piece of music); emotional contagion (the listener's response and understanding of expressive acoustic cues); visual imagery (an emotion is evoked because of the close relation between the music and the listener's mental images); episodic memory (listening to music evokes a specific visual memory that can involve different kinds of life events); and musical expectancy (emotion is induced because a specific feature of the music violates, delays, or confirms expectations on the continuation of the music). In line with recent comprehensive models for visual art and other aesthetic experiences (Pelowski, Markey, Forster, \& Gerger, 2017), Brattico and colleagues (2013, p. 6) propose that brainstem reflexes are accompanied by "their conscious categorical attribution as discrete emotions, and finally leading to the outcomes of aesthetic emotions, aesthetic judgments, and attitudes". Basic emotions, such as joy or sadness, can be expressed by the sender (the composer and/or performer), perceived by the receiver (the listener), and possibly felt by the receiver as well as the sender. Furthermore, besides these emotions lasting just few seconds in time, music can also alter the listener's mood or background emotional state. Mood is less intense than an emotional episode, but nevertheless tinges daily life and affects behaviour (Parkinson, Totterdell, Briner, \& Reynolds, 1996). Mood alteration or regulation is often considered a central function of musical activities and can also lead to positive health outcomes. By contrast, the alteration of emotions associated with listening to music produces only immediate rewards (Gabrielsson, 1993).

Another outcome of musical experience is conscious liking. This refers to the voluntary, often verbal, evaluative judgement of the whole musical event in relation to its emotional goals 
and to its success or failure in inducing discrete and aesthetic emotions, particularly enjoyment. When liking for a piece of music, or a set of pieces sharing similar stylistic features, is stable over time, it is known as preference. Preference becomes taste when preference is not only for separate pieces of music but also for their style, as a whole, in the context of social and cultural factors (Brattico et al., 2013).

Aesthetic musical experiences also depend on external and internal factors, such as environment, peers and setting (external context), and mood, attention, attitudes, intentionality and expertise (internal context) (Brattico et al., 2013). These top-down modulatory factors are independent of the physical musical features and vary substantially among individuals. According to Mencke, Omigie, Wald-Fuhrmann, and Brattico (2018), this is exemplified by the aesthetic enjoyment of atonal music characterized, for many, by sensory dissonance and unpredictability. Nevertheless, it is enjoyed by a group of enthusiastic listeners. On the one hand, this may be due to the mere exposure effect, the positive effect of prior listening on liking (Montoya, Horton, Vevea, Citkowicz, \& Lauber, 2017; Zajonc, 1968), or cognitive mastery, that is, the ability to increase appreciation of a musical piece using cognitive constructs such as information regarding the piece or the artist. On the other hand, the intrinsic uncertainty of atonal music, which makes it hard for listeners to form predictive models, might constitute a source of pleasure for individuals with more open and novelty-seeking personality traits (Mencke et al., 2018).

Studies of liking and preference for music have been reviewed by Hargreaves and North (2010). Many empirical studies of listeners' and performers' aesthetic experiences in relation to music have been undertaken in recent years but have not yet been reviewed systematically or comprehensively. Meanwhile, few experimental or review studies of the neural determinants of musical aesthetic experience have seen the light of day (Brattico et al., 2013; Reybrouck, Vuust, $\&$ Brattico, 2018). The following section provides an overview of relevant findings.

\section{Neural mechanisms underlying aesthetic experiences and pleasure}

Neuroscientific studies show that emotional responses to sounds originate from several brain structures involving cortical as well as subcortical limbic and paralimbic structures, such as the ventral striatum, including the nucleus accumbens, the lateral and ventromedial orbitofrontal cortex and the anterior cingulate cortex. They also originate from the regions responsible for reactions of the autonomic nervous system and the body, such as the insula, the thalamus, the hypothalamus and the brainstem nuclei.

In the brain, negative emotions such as fear are physiological states controlled unconsciously by subcortical circuits involving the amygdala, which mediates between threatening stimuli and defence responses (Blood \& Zatorre, 2001; Koelsch, 2014; LeDoux, 2017). Thus, the amygdala plays a very important role in recognizing and perceiving sad (Liégeois-Chauvel et al., 2014), scary, dissonant and unpredictable music (Brattico \& Pearce, 2013; Brattico et al., 2013). Even sadness expressed in songs elicits increased amygdala activity (Brattico et al., 2011). The neural response of the amygdala to both fear and unpleasantness might be related to acoustic cues that are common to both emotions. For instance, both human screams, which function as natural alarm signals in human communication and are a major component in certain styles of rock music, and dissonant chords are characterized by high roughness, originating from slow sound modulation rates between $\sim 30$ and $150 \mathrm{~Hz}$. Recent neuroimaging studies show that rough temporal modulations of sounds such as screaming, scratching, or breaking glass selectively activate the primary auditory cortex and the amygdala (Arnal, Flinker, Kleinschmidt, Giraud, \& Poeppel, 2015; Kumar, Forster, Bailey, \& Griffiths, 2008), 
unlike neutral sounds. It can thus be proposed that the amygdala contains the neural coding mechanisms enabling people to respond quickly to acoustic cues related to salience in the auditory environment and potential danger.

Positive emotions, including happiness expressed and induced by music and pleasure, are associated with activity in the auditory cortex and in several limbic and paralimbic regions. Among them, the nucleus accumbens, which is part of the ventral striatum and both anatomically and functionally connected to orbitofrontal cortices via dopaminergic neurotransmission, plays a key role in processing intense pleasure evoked by music (including chills; Alluri et al., 2015; Salimpoor et al., 2013; Salimpoor, Benovoy, Larcher, Dagher, \& Zatorre, 2011). Notably, dopaminergic neurotransmission in the striatum occurs even in response to cues predicting spatially distant goals and rewards in animal models (Howe, Tierney, Sandberg, Phillips, \& Graybiel, 2013). Blood and Zatorre (2001) employed positron emission tomography (PET) to conduct the first neuroimaging study on the neural correlates of the intense aesthetic enjoyment of music. The experiment consisted of a listening task employing self-selected classical instrumental music inducing chills (goose bumps and shivers down the spine) as opposed to music selected by other participants. Significant positive correlations between metabolic brain signal and the intensity of chills, experienced only during self-selected music, were observed in the ventral striatum, midbrain, thalamus and orbitofrontal cortex (regions previously found active in relation to pleasure or euphoria derived from sex, for example, and food), as well as in the supplementary motor area and cerebellum. In turn, negative correlations evidenced activity in the amygdala and surrounding regions as well as in the ventral medial prefrontal cortex and visual cortex.

Although music-induced aesthetic emotional experiences are usually positive, it should not be inferred that they are induced only by "merry" music. Since there is some overlap between the neural correlates of music-induced pleasure and displeasure, on the one hand, and those of "sad" and "happy" emotions in music, on the other, sad music has the ability to induce positive aesthetic emotions of enjoyment (Brattico et al., 2016; Vuoskoski \& Eerola, 2017; Eerola, Vuoskoski, Peltola, Putkinen, \& Schäfer, 2018; Taruffi \& Koelsch, 2014). Brattico (2015, p. 304) defines the phenomenon as "the paradox of a stimulus which begets a positive aesthetic response despite its intrinsic negative properties”. In a recent fMRI study (Brattico et al., 2016), musicians and non-musicians listened to 18 -second self-selected musical excerpts they reported as liked/happy, liked/sad, disliked/happy, or disliked/sad. The results revealed that the cortical temporofrontal structures activated in response to the perception of sadness or happiness in music were distinct from those activated in response to aesthetic evaluation, localized in deep subcortical centres governing arousal and the autonomic nervous system. Based on these and other findings, Brattico and colleagues (2016, p. 18) proposed that conscious liking depends on "an implicit appraisal of the general affective state induced by it".

Several findings converge to suggest that prefrontal and parietal brain regions have to be activated for listeners to experience cognitive types of emotion in relation to music, such as being able to recognize a melody as "sad" or "happy", or liking music because it complies with stylistic conventions (Brattico et al., 2011; Bogert et al., 2016; Brattico et al., 2016; Koelsch, 2014; Koelsch, 2010; Zatorre \& Salimpoor, 2013; Salimpoor \& Zatorre, 2013; Salimpoor et al., 2015; Reybrouck \& Brattico, 2015). These areas of the brain might then enable "conscious reflection and categorical labelling of the bodily changes associated with a stimulus or an event" (Brattico et al., 2013, p. 8).

The interplay between brain structures related to hedonic responses to music and those related to cognitive processing and evaluative judgements is evidenced in neuroimaging studies of functional connectivity, namely the temporal pairwise correlation between signals coming 
from distinct brain locations. For instance, Salimpoor and colleagues (2013) identified the neuronal communication between the main structures active during intense musical pleasure. For example, when participants were invited to "purchase" 30-second excerpts of music for variable sums of money, activity and connectivity in the dorsal (caudate) and ventral (right nucleus accumbens) striatum accounted for both reported music liking and the amount of money people were willing to spend, the nucleus accumbens being the main source of variation. Moreover, when the music was desirable, the nucleus accumbens increased communication with cortical and subcortical areas such as the amygdala, superior temporal gyrus and inferior prefrontal cortex. While these experiments studied connections within a single brain region, a pioneering study using network science methods looked at reciprocal connections between all regions of the brain while participants were listening to liked familiar pieces and novel pieces of music in a favourite musical genre (Wilkins, Hodges, Laurienti, Steen, \& Burdette, 2014; for a comprehensive review of studies on functional connectivity during aesthetic music listening, see Reybrouck et al., 2018). The results showed that the medial and parietal regions of the brain became more connected when listening to preferred music, and that listening to favourite songs altered the connectivity between auditory brain areas and the hippocampus, a region responsible for memory and social-emotional consolidation (Koelsch, 2014; Burunat, Alluri, Toiviainen, Numminen, \& Brattico, 2014).

Overall, these findings of increased connections between auditory cortex and striatal reward centres during pleasurable aesthetic experiences of listening to music, derived from studies using neuroimaging techniques, were obtained from experiments involving acoustically diverse music, self-selected by the participants. This suggests that music-induced pleasure depends not only on the features of the stimulus but also on the listener and context (Liu et al., 2017; Hargreaves \& North, 2010). Moreover, aesthetic experiences can be incidental and spontaneous, as demonstrated in a study in which reward-related striatal and orbitofrontal structures were more strongly connected during the spontaneous enjoyment of music excerpts than when participants had expressed conscious liking or disliking (Liu et al., 2017).

Other studies of functional connectivity reveal a relationship between the default mode network (DMN) and aesthetic music listening (Reybrouck et al., 2018; Alluri et al., 2017). The DMN consists of interconnected brain regions such as the precuneus, ventromedial prefrontal cortex, dorsolateral prefrontal cortex, posterior cingulate cortex, and some regions of the parietal cortex and the medial temporal lobe, which continue to fluctuate in synchrony with each other, monitoring the sensory environment, even while participants are awake but in a resting state. Activation of the DMN relates to processes of self-estimation and mindwandering, self-referential thoughts and imagining the future (Mitra \& Raichle, 2018). It seems to be active especially when listeners are exposed to sad, rather than happy music (Taruffi, Pehrs, Skouras, \& Koelsch, 2017). Hence, a possible function of the DMN could be to facilitate aesthetic contemplation and relaxation while listening to favourite music (Reybrouck et al., 2018).

Another important aesthetic response, namely the judgement of aesthetic value, has only rarely been targeted by neuroscientific investigation, possibly due to the complex nature of these judgements and the lack of an agreed conceptual definition (for a review on the topic, see Brattico et al., 2013). Even less research has been undertaken to attempt an understanding of the neural mechanisms permitting intentionality to be ascribed to music, which some deem an essential aspect of aesthetic experience (Snapper et al., 2015; Brattico, Brattico, \& Vuust, 2017). Initial results highlighted the role of the medial orbitofrontal cortex in the judgement of musical beauty (Ishizu \& Zeki, 2011) and of theory-of-mind (TOM) regions (anterior medial frontal cortex, superior temporal sulcus and temporal poles) in intention attribution (Steinbeis 
\& Koelsch, 2009). On a more positive note, the field of empirical aesthetics of music is slowly maturing, with several attempts to reach consensus (Schubert \& North, 2016; Brattico, 2016; Juslin, 2013a, 2013b) and a growing interest in psychobiological research (Brattico, in press).

\section{The long-term impact of aesthetic experiences of music on the brain}

Brain plasticity is the remarkable ability of the brain to respond and adapt to new demands and conditions imposed by the external world (Miendlarzewska \& Trost, 2013). For musicians, the external world is represented by their long-term commitment to training for several hours each day over many years, the instruments they play, their responses to sounds, actions and their own bodily sensations, and the rewards they gain from internal and external feedback from their own enjoyment and from listeners' aesthetic reactions. These all contribute to the musician's long-term aesthetic experience of music. They encompass perceptual, cognitive, affective, proprioceptive, evaluative and social functions and have been shown to influence the physiology and anatomy of the brain.

Several neuroimaging studies have found enlarged volumes in musicians' brain areas related to auditory perception, such as Heschl's gyrus and the superior temporal gyrus, and fine motor actions, such as the premotor and motor cortex (Schlaug, 2003; Wan \& Schlaug, 2013; Kraus \& White-Schwoch, 2017). Both the cerebellum, involved with fine-grained control of movements, and the corpus callosum, the bundle of white matter fibres connecting the two cerebral hemispheres, are consistently larger in musicians than in non-musicians, possibly because of the bimanual coordination demands of playing a musical instrument (Burunat et al., 2015). The results of longitudinal studies involving the analysis of data from participants - typically children - before and after engaging in a music training programme (Hyde et al., 2009; Habibi, Cahn, Damasio, \& Damasio, 2016; Putkinen, Tervaniemi, Saarikivi, Ojala, \& Huotilainen, 2014) confirms that musical training is the main force driving increases in brain volume.

Moreover, musicians represent an ideal population for studying the plasticity of limbic brain functions, as they have spent years learning to predict expressive sounds and refine their ability to produce music that conveys emotions and induces pleasure. Musical training thus might be related to changes in the structures of and connectivity within the limbic system. For instance, Brattico and colleagues (2016) found that professional instrumental musicians who had undertaken many years of regular daily practice showed increased regional neural activity in the limbic system and areas related to salience processing such as the insula and the anterior cingulate cortex, in comparison with non-musicians, while listening to favourite expressive music in musicians. Similar findings of increased temporal-limbic responses were obtained, using electroencephalographic (EEG) methods, from pianists listening to excerpts of novel expressive music with incongruous endings (James, Britz, Vuilleumier, Hauert, \& Michel, 2008), and using fMRI measurements, from musically trained participants listening to recordings of expressive and inexpressive performances of a Chopin étude (Chapin, Jantzen, Kelso, Steinberg, \& Large, 2010). Specifically, in the latter study, activity in the action observation system (premotor cortex, supplementary motor area, inferior parietal lobe) of experienced participants was correlated with the temporal dynamics of the expressive performance. In line with this, it is proposed that music evokes emotions by means of an empathic process that recruits the action observation system with the limbic system via the insula to interiorize the expressive gestures of others, rather than cognitive interpretation (Molnar-Szakacs \& Overy, 2006; Juslin \& Västfjäll, 2008; Koelsch, 2014). 
Even connectivity between limbic structures and the rest of the brain depends on musical training. In an fMRI study by Alluri and colleagues (2015), musicians but not non-musicians, when listening to three 8-minute-long pieces of music in different styles, displayed increased connections from brain structures controlling emotional and rewarding experiences, such as the bilateral amygdala, hippocampus and left nucleus accumbens, and the rest of the brain.

In all the studies mentioned above, the differences between musicians and non-musicians were assessed by questionnaires investigating self-reported hours of playing, practising and listening. Theoretical knowledge of music was not assessed, nor were participants asked about the financial and/or social rewards they gained from making music. The impact of theoretical knowledge and rewards on limbic system responses could be addressed by studying other professional musicians who are not primarily instrumentalists, such as record producers or DJs. In sum, it is reasonable to suppose, even on the basis of limited evidence, that many years' experience of aesthetic listening affects the regions of the brain, and the connections between them, that control emotional perception and subjective feelings.

\section{The impact of aesthetic musical experiences on disease}

There is an ancient presupposition that the prolonged and systematic practice of aesthetic listening to music has beneficial effects on health and disease. This is supported by the evidence of increased neural activity and enlarged brain regions related to perception, action and emotion after repeated aesthetic experiences involving listening to and/or performing music in healthy individuals (Thaut, 2015; Thaut \& Wheeler, 1993; MacDonald, Kreutz, \& Mitchell, 2013; Altenmüller \& Schlaug, 2015; Pauwels, Volterrani, Mariani, \& Kostkiewics, 2014). Such experiences may represent a means for personal empowerment by equipping individuals with tools for coping in the world and enhancing the basic conditions of human life. Indeed, the therapeutic effects of aesthetic pleasure derived from music have been documented in several pathological conditions, such as stroke, chronic pain, anxiety, depression and schizophrenia. Here, we will provide a brief excursus on some of the most striking applications of listening to emotionand pleasure-inducing music for the daily empowerment of individuals with pathological symptoms.

One example of the clinical efficacy of exposure to favourite music can be found in research on Alzheimer's disease (AD), the most common form of dementia due to a progressive neurodegenerative disorder. AD damages episodic memory "with more variable impairment of semantic memory and relative preservation of procedural memory" (Clark \& Warren, 2015, p. 2123). Several studies have provided evidence of the benefits of interventions involving music for AD patients (Peck, Girard, Russo, \& Fiocco, 2016; Matrone \& Brattico, 2015). Guided music listening sessions, in particular, enhance arousal, reduce anxiety and increase cognitive functions such as attention, memory (both autobiographical memory and involuntary recall) and speech (enhanced verbal narration). The findings of most studies indicate that even when patients are in the advanced stages of $\mathrm{AD}$, their musical memory is relatively preserved (Matrone \& Brattico, 2015; Peck et al., 2016) and they can still experience enjoyment when listening to music. However, Petrovsky, Cacchione, and George (2015) argue, in their review of the literature on anxiety and depression in older adults with mild dementia, that the majority of the studies they reviewed lacked methodological rigour and therefore produced inconsistent support for interventions involving music, including music therapy, as an effective treatment for anxiety and depression. Future studies of patients with dementia should clearly use better and more consistent methodological approaches. Nevertheless, Petrovsky and colleagues (2015) note the 
positive results that have been reported, suggesting that music has the potential to be an important therapeutic intervention for alleviating patients' psychological and behavioural symptoms.

Some researchers suggest that it is not the music but the music-induced emotion that enhances long-term episodic recall, largely in the form of involuntary memories rather than recent autobiographical memories. These are important for the feelings of material, social and spiritual self-identity evoked mostly by familiar music (Peck et al., 2016). A study by Särkämö and colleagues (2014) found that elderly patients with mild dementia who regularly took part in musical activities (singing and listening to familiar songs) obtained social, emotional and cognitive benefits. Their mood improved and their general cognitive abilities, orientation, attention, executive function and remote episodic memory were enhanced or at least maintained. They also scored higher on the quality of life (QOL) scale. Singing in the absence of other musical activities enhanced short-term and working memory; it also improved the wellbeing of family members who acted as caregivers. Generally, studies of the effects on people with mild AD of music making and listening suggest that it can enhance memory by activating autobiographical recall and its associated emotions.

Other promising evidence as to how aesthetic music listening might empower individuals in challenging life conditions comes from studies on pain, both transient and chronic. Behavioural experiments have repeatedly shown that music can alleviate the intensity and unpleasantness of laboratory-induced (e.g., cold-pressor) pain, especially when chosen by the participant rather than the experimenter (Mitchell \& MacDonald, 2006). Results are less clear in relation to pain during surgical procedures (MacDonald et al., 2003). For instance, patients who had undergone a minor surgical procedure to the foot and listened to self-selected music for at least 45 minutes post-surgery reported experiencing less anxiety than controls who did not listen to music. However, the two groups did not differ on measures of pain perception.

Initial findings in relation to the effects of listening to music on chronic pain have been obtained from patients with fibromyalgia. This is a syndrome characterized by diffuse multifocal pain accompanied by fatigue, sleep disturbances, depression, cognitive and memory issues, alterations in sensory processing, and significant limitations in functional mobility or the ability to perform goal-directed movements. A behavioural study demonstrated that patients' functional mobility improved significantly when they listened to 10 minutes of slow self-chosen music before undertaking a "timed-up and go" task instead of a control auditory sequence, to a degree that correlated with the unpleasantness of the pain they perceived (Garza-Villarreal et al., 2014). In a subsequent study (Garza-Villarreal et al., 2015), neuroimaging data during rest while lying in the fMRI scanner were obtained from the same patients after the two listening conditions. Results showed that low frequency fluctuations in the angular gyrus, a core centre of the DMN, had increased amplitude only after the patients had listened to their favourite music, but not after they had heard the control sequence. Similarly, higher connectivity with the pain modulatory network was observed in patients after listening to their favourite music, but not the control sequence. There is thus an accumulation of evidence to support the hypothesis that listening to pleasurable music can reduce acute and even chronic pain.

Overall, the findings of these and several other studies of the effects of enjoying music on a variety of clinical conditions provide compelling evidence of its benefits for health. They should encourage healthy individuals, patients and caregivers to embrace musical activities in their daily routines, particularly listening to favourite music, for personal empowerment ranging from hedonia (immediate pleasure) to eudaimonia (subjective wellbeing or the sense of a life well lived) (Stark et al., 2018). 


\section{Neural mechanisms responsible for aesthetic empowerment through music}

Inspired by a proposal of Peck and colleagues (2016), we suggest four putative neural biological mechanisms, shown in Figure 1, which might be responsible for the beneficial effects of aesthetic musical experiences, such as listening to favourite music, playing an instrument, or singing, on personal empowerment. These beneficial effects include improved mood, focused attention, the facilitation of learning, and the enhancement of memory.

The first is a neurotransmitter mechanism that has a direct impact on mood and is related to the activation of mesolimbic dopaminergic pathways, which branch off from the ventral tegmental area and pass by the ventral striatum (nucleus accumbens). Neuroscientific research provides evidence that music listening in health and even disease enhances mood and motivation. This seems to be achieved by strengthening dopaminergic activity within reward circuits, which results in stimulation for learning new information and "enhances emotional processing through autonomic activation while attaching a cognitive aspect of reward to the experience” (Peck et al., 2016, p. 953). According to Kringelbach and Berridge (2017), this pleasure system incorporates a simpler and more spontaneous core liking reaction generated by mesolimbic hotspots situated in the ventral striatum, in the insula and the orbitofrontal cortex, which are activated in the presence of a pleasurable stimulus even when a conscious feeling of liking does not occur. This core hedonic response is accompanied by "wanting" or incentive salience during the appetitive phase. The neural correlates of incentive salience consist of dopaminergic neurotransmission between several brain regions, including the anterior cingulate cortex, the mid-anterior part of the orbitofrontal cortex, the insula and ventromedial regions of the prefrontal cortex. Learning processes are also part of this pleasure cycle. Music triggers all of these pleasure processes daily, hence contributing to eudaimonia.

The second physiological mechanism, closely linked to the first one, is sympathetic arousal. Since emotional responses to music rely on dopaminergic neurotransmission and pathways that are anatomically connected with the hypothalamus, musical activities are capable of changing the sympathetic and parasympathetic activity of the autonomic nervous system (ANS) such that heart rate, blood pressure, and processes such as respiration are increased or attenuated. These physiological responses, in turn, can help reduce anxiety and improve attention and memory. In this way musical activities reduce anxiety, improve attention and memory and thereby regulate listeners' mood; "Music initiates brainstem responses that, in turn, regulate heart rate, pulse, blood pressure, body temperature, skin conductance, and muscle tension, partly via noradrenergic neurons that regulate cholinergic and dopaminergic neurotransmission" (Chanda \& Levitin, 2013, p. 186).

The third mechanism proposed here is the large-scale brain network known as the DMN. Although there is still much to learn and understand about the DMN, many neuroscientific studies imply that it may play an important role in memory and "self-referential processes such as introspection and autobiographical memory retrieval” (Peck et al., 2016, p. 954). Furthermore, DMN connectivity stimulated by familiar music listening in healthy participants (e.g., Wilkins et al., 2014) may contribute to memory and enhance attention. Finally, since many key regions of the pleasure network are also part of the DMN, it may be important for personal empowerment by turning hedonic pleasure into deep personal happiness or eudaimonia (Kringelbach \& Berridge, 2017, p. 195). Related to this, the subjective improvement in mood of patients who have recovered from depression is marked neurally by changes in the connectivity between the DMN and striatal structures of the brain (Andreescu et al., 2013). 


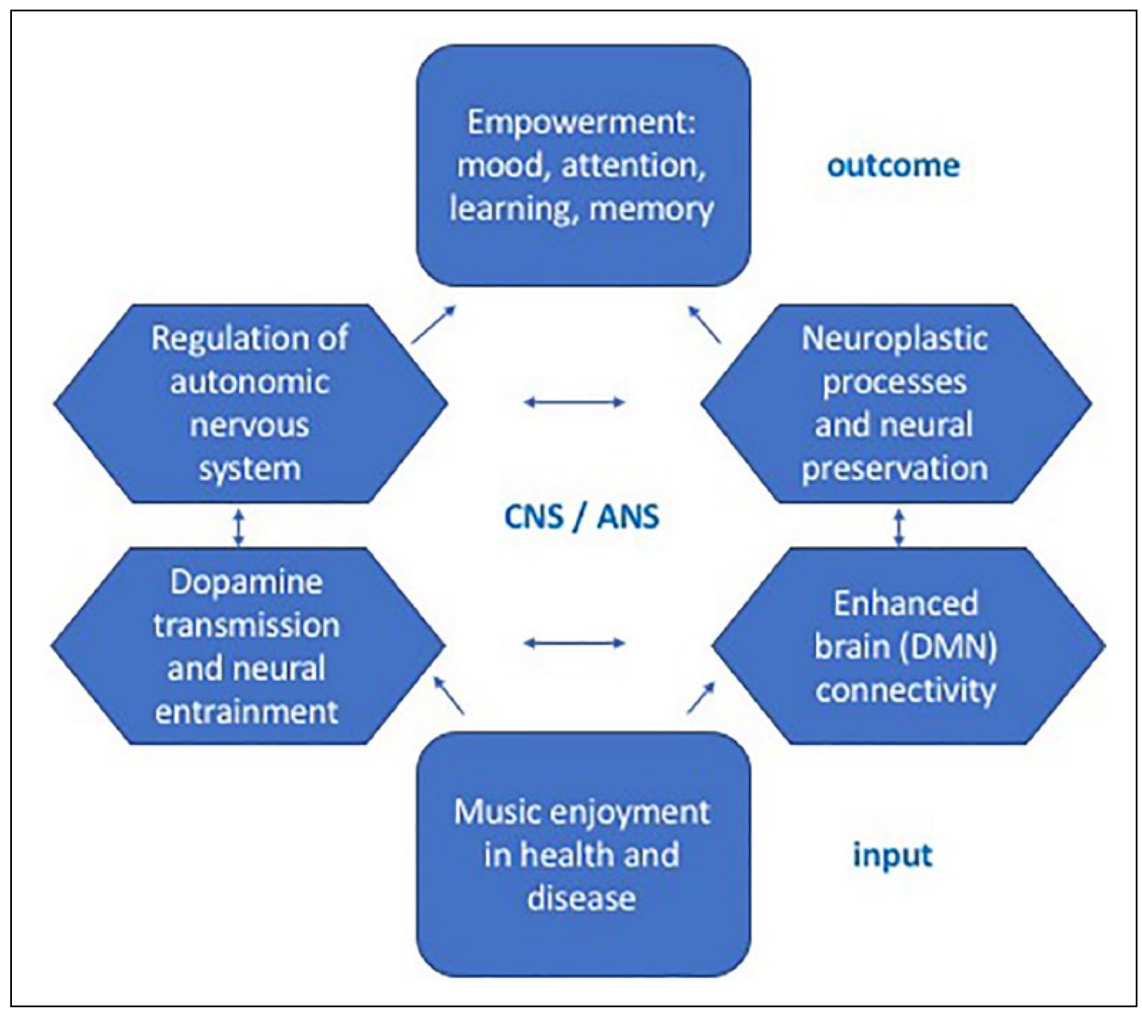

Figure I. Schematic representation of the putative biological mechanisms that might explain the empowering effect of aesthetic listening to music.

A fourth mechanism predicting an association between enjoyment of music and its empowering impact on behaviour in health and disease relates to the neuroplastic processes that occur in the learning phase of the pleasure cycle (Kringelbach \& Berridge, 2017) after repeated music listening or playing. For instance, in a study of schizophrenia patients who listened peacefully to a sonata by Mozart for 30 minutes every day for a month, an increase in resting-state functional connectivity was observed from insula regions to cingulate regions, in particular. Accumulating evidence also points to the protective effect of music, especially music making, on neural degeneration resulting not only from the natural non-pathological process of aging but potentially also from neurodegenerative diseases. This protective effect might be mediated by music-induced neuroplastic processes reinforced by the brain-derived neurotrophic factor (BDNF). BDNF is a protein present in several parts of the human nervous system, and is known to maintain the dendritic complexity and spine density of neuronal cells in selected brain centres and in the cochlear afferents of the inner ear (Singer, Panford-Walsh, \& Knipper, 2014). Furthermore, a recent study using anatomical MR images and machine learning algorithms computed the discrepancy in years between chronological age and the "age of the brain" (Rogenmoser, Kernbach, Schlaug, \& Gaser, 2018). Amateur musicians had "younger" brains than non-musicians, and even professional musicians, suggesting that the greatest benefits come from engaging in music making as one of several activities rather than just one. There has been an accumulation of evidence in recent years for neuroplastic changes in patients with 
several clinical conditions following prolonged listening to favourite music (Särkämö, 2018; Särkämö, Ripollés, et al., 2014), engagement in musical activities (MacDonald et al., 2013; Altenmüller \& Schlaug, 2015), and even short periods of singing in a choir (Dubinsky, Nespoli, \& Russo, 2017; Kreutz, Bongard, Rohrmann, Hodapp, \& Grebe, 2004). These neuroplastic changes underlie behavioural benefits, such as the preservation and rehabilitation of cognitive skills as well as mood improvement in elderly people and those with dementia, or who have suffered a stroke (Särkämö \& Sihvonen, 2018; Särkämö, 2018).

\section{Idiosyncrasy in aesthetic responses to music and the importance of accounting for it}

When considering the impact of music on human life, it is relevant to consider that, although music can generally be described as a universal means of communication, not everyone experiences music-induced aesthetic emotions or pleasure in response to the same stimuli (Huron, 2011) or at the same level of intensity. This can be explained by the variability of individual factors, such as musical aptitude, listening biography, engagement, sensitivity to musical rewards, and so on. These factors can be categorized as biological or cultural, depending on whether they are related to stable biological markers such as neuroanatomical structures or genes, or if they can be attributed to the influence of environmental exposure, which varies from one context to another and over time.

In previous sections we have explored the influence of exposure and familiarity. Now we highlight some of the biological factors that affect aesthetic responses to music. As pointed out by Brattico (2015, p. 313), "low-level sensory processes already vary between individuals and within the same individual in quite a stable way due to inborn, genetic predispositions for micro- and micro-structural physiological properties of critical brain regions such as the auditory cortex and amygdala, which are both crucial for musical emotions". Indeed, the first biological source of variability lies in the genes responsible for the auditory skills most relevant to music, such as the ability to discriminate between pitch frequencies and time durations. These abilities are thought to be heritable and transmitted genetically (Gingras, Honing, Peretz, Trainor, \& Fisher, 2015; Drayna, Manichaikul, de Lange, Snieder, \& Spector, 2001; Peretz \& Vuvan, 2017; Peretz, Cummings, \& Dubé, 2007) as, like congenital amusia, they are often shared by members of the same family. A set of candidate genes has been proposed by the authors of recent studies who used listening tests designed to assess musical abilities in the general population, one of which was designed by Seashore (1911) and consists of pairwise comparisons of the frequency and duration of sounds. Performance on these listening tests is associated with regions of the DNA that contain gene variations affecting the development and functioning of auditory pathways and neurocognitive processes (Järvelä, 2018; Ukkola-Vuoti et al., 2013). Oikkonen, Onkamo, Järvelä, and Kanduri (2016) carried out a meta-analysis of molecular studies related to auditory music-related abilities, listening and sound production in both humans and in animal species, collecting a total of 7895 relevant genes and biomarkers from 105 different studies. Of the most plausible candidate genes, EGR1, FOS, ARC, BDNF and DUSP1, several are known to be regulated by sensory and motor behaviour in the brain, and they also have crucial roles in vocal learning and sound production in several animal species such as zebra finches and other songbirds. Furthermore, CDK5 signalling pathway-related genes, related to cognitive functions including learning and memory, were found to be enriched in musically able individuals; the authors suggest that this finding might provide a molecular basis for those cognitive processes involved in musical activities. 
Another potential source for the biological origins of individual variability in aesthetic reactions to music is dopaminergic neurotransmission and its pathways. Sachs, Ellis, Schlaug, and Loui (2016) investigated individual differences in responses to musical stimuli by using diffusion tensor imaging (DTI) to measure structural connectivity and psychophysiological responses. Participants were grouped on the basis of an online survey into a) those who reported consistently experiencing music-induced chills, and b) those who did not. The "chill" group experienced chills during a listening task in a laboratory setting and showed higher white matter volume in tracts connecting the superior temporal gyrus with the insula and the medial prefrontal cortex bilaterally than did the "no-chill" group (Sachs et al., 2016). Individual variations in functional fMRI connectivity between the auditory cortex and the ventral striatum were also found in relation to musical aesthetic sensitivity, as assessed with the Barcelona Musical Reward Questionnaire (Martínez-Molina, Mas-Herrero, Rodríguez-Fornells, Zatorre, \& Marco-Pallarés, 2016). These variations might derive from congenital mutations of dopamine receptor genes, which are relatively common in the general population. In a study by Quarto and colleagues (2017), where an imaging genetic approach was used for the first time in the neuroscientific literature on music, the genetic variability of dopamine D2 type receptors (DRD2 genotype) modulated the effects of background music and noise on mood states, and on prefrontal and striatal activity in response to the implicit processing of visual emotions represented by facial expressions. Overall, the findings outlined above suggest that there is a biological source of variability behind music-induced emotion processes.

\section{Conclusions}

In this article we have argued that musical activities inducing positive aesthetic responses, specifically enjoyment and liking, empower the individual in health and disease by improving mood, cognitive functions, happiness and quality of life. Studies investigating aesthetic experiences of music are still scarce, and the resulting picture tends to be rather unclear. Several issues remain to be overcome in research investigating the essence of musical aesthetic experiences. However, what emerges as a constant leitmotiv in this line of research is the efficacy of musical enjoyment for making a positive impact on human life, both in health and disease.

Future studies on aesthetic empowerment through music making and listening should move away from the artificial laboratory settings towards more ecological ones (Burunat et al., 2016; Alluri et al., 2015) in which participants can experience musical activities as they do in their everyday lives. The findings of such research could be even more compelling, enabling recommendations to be made as to the optimal daily use of music even in the most challenging life situations.

\section{Funding}

The author(s) disclosed receipt of the following financial support for the research, authorship, and/or publication of this article: Center for Music in the Brain (MIB) is funded by the Danish National Research Foundation (DNRF 117).

\section{References}

Alluri, V., Brattico, E., Toiviainen, P., Burunat, I., Bogert, B., Numminen, J., \& Kliuchko, M. (2015). Musical expertise modulates functional connectivity of limbic regions during continuous music listening. Psychomusicology: Music, Mind, and Brain, 25(4), 443-454.

Alluri, V., Toiviainen, P., Burunat, I., Kliuchko, M., Vuust, P., \& Brattico, E. (2017). Connectivity patterns during music listening: Evidence for action-based processing in musicians. Human Brain Mapping, 38(6), 2955-2970. 
Altenmüller, E., \& Schlaug, G. (2015). Chapter 12 - Apollo's gift: New aspects of neurologic music therapy. In E. Altenmüller, S. Finger, \& F. Boller (Eds.), Music, Neurology, and Neuroscience: Evolution, the Musical Brain, Medical Conditions, and Therapies (pp. 237-252). Amsterdam, The Netherlands: Elsevier.

Andreescu, C., Tudorascu, D. L., Butters, M. A., Tamburo, E., Patel, M., Price, J., ... Aizenstein, H. (2013). Resting state functional connectivity and treatment response in late-life depression. Psychiatry Research, 214(3), 313-321.

Arnal, L. H., Flinker, A., Kleinschmidt, A., Giraud, A.-L., \& Poeppel, D. (2015). Human screams occupy a privileged niche in the communication soundscape. Current Biology: CB, 25(15), 2051-2056.

Blood, A. J., \& Zatorre, R. J. (2001). Intensely pleasurable responses to music correlate with activity in brain regions implicated in reward and emotion. Proceedings of the National Academy of Sciences, 98(20), 11818-11823.

Bloom, P. (1996). Intention, history, and artifact concepts. Cognition, 60(1), 1-29.

Bogert, B., Numminen-Kontti, T., Gold, B., Sams, M., Numminen, J., Burunat, I., ... Brattico, E. (2016). Hidden sources of joy, fear, and sadness: Explicit versus implicit neural processing of musical emotions. Neuropsychologia, 89, 393-402.

Brattico, E. (2015). From pleasure to liking and back: Bottom-up and top-down neural routes to the aesthetic enjoyment of music. In Art, Aesthetics, and the Brain (pp. 303-318).

Brattico, E. (2016). Commentary on 'Aesthetic experience explained by the affect-space framework' by E. Schubert, A. C. North, \& D. J. Hargreaves. Empirical Musicology Review: EMR, 11(3/4).

Brattico, E. (in press). The neuroaesthetics of music: A research agenda coming of age. In M. Thaut \& D. Hodges (Eds.), The Oxford handbook of music and the brain. Oxford, UK; New York, NY: Oxford University Press.

Brattico, E., Alluri, V., Bogert, B., Jacobsen, T., Vartiainen, N., Nieminen, S., \& Tervaniemi, M. (2011). A functional MRI study of happy and sad emotions in music with and without lyrics. Frontiers in Psychology, 2, 308.

Brattico, E., Bogert, B., Alluri, V., Tervaniemi, M., Eerola, T., \& Jacobsen, T. (2016). It's sad but I like it: The neural dissociation between musical emotions and liking in experts and laypersons. Frontiers in Human Neuroscience, 9. DOI: 10.3389/fnhum.2015.00676

Brattico, E., Bogert, B., \& Jacobsen, T. (2013). Toward a neural chronometry for the aesthetic experience of music. Frontiers in Psychology, 4, 206. DOI: 10.3389/fpsyg.2013.00206

Brattico, E., Brattico, P., \& Vuust, P. (2017). The forgotten artist: Why to consider intentions and interaction in a model of aesthetic experience: Comment on 'Move me, astonish me ... delight my eyes and brain: The Vienna Integrated Model of top-down and bottom-up processes in Art Perception (VIMAP) and corresponding affective, evaluative, and neurophysiological correlates' by Matthew Pelowski et al. Physics of Life Reviews, 21, 128-130.

Brattico, E., \& Pearce, M. (2013). The neuroaesthetics of music. Psychology of Aesthetics, Creativity, and the Arts, 7(1), 48-61.

Burunat, I., Alluri, V., Toiviainen, P., Numminen, J., \& Brattico, E. (2014). Dynamics of brain activity underlying working memory for music in a naturalistic condition. Cortex: A Journal Devoted to the Study of the Nervous System and Behavior, 57, 254-269.

Burunat, I., Brattico, E., Puoliväli, T., Ristaniemi, T., Sams, M., \& Toiviainen, P. (2015). Action in perception: Prominent visuo-motor functional symmetry in musicians during music listening. PloS One, 10(9), e0138238.

Burunat, I., Toiviainen, P., Alluri, V., Bogert, B., Ristaniemi, T., Sams, M., \& Brattico, E. (2016). The reliability of continuous brain responses during naturalistic listening to music. NeuroImage, 124(Pt A), 224-231.

Chanda, M. L., \& Levitin, D. J. (2013). The neurochemistry of music. Trends in Cognitive Sciences, 17(4), $179-193$.

Chapin, H., Jantzen, K., Kelso, J. A. S., Steinberg, F., \& Large, E. (2010). Dynamic emotional and neural responses to music depend on performance expression and listener experience. PloS One, 5(12), e13812. Retrieved from https://doi.org/10.1371/journal.pone.0013812 
Clark, C. N., \& Warren, J. D. (2015). Music, memory and mechanisms in Alzheimer's disease. Brain: A Journal of Neurology, 138(Pt 8), 2122-2125.

Drayna, D., Manichaikul, A., de Lange, M., Snieder, H., \& Spector, T. (2001). Genetic correlates of musical pitch recognition in humans. Science, 291(5510), 1969-1972.

Dubinsky, E., Nespoli, G., \& Russo, F. (2017). Effects of short-term choir participation on auditory perception in older adults. Canadian Acoustics, 45(3). Retrieved from https://jcaa.caa-aca.ca/index.php/ jcaa/article/view/3143

Eerola, T., Vuoskoski, J. K., Peltola, H.-R., Putkinen, V., \& Schäfer, K. (2018). An integrative review of the enjoyment of sadness associated with music. Physics of Life Reviews, 25, 100-121.

Fitch, W. T. (2015). Four principles of bio-musicology. Philosophical Transactions of the Royal Society of London. Series B, Biological Sciences, 370(1664), 20140091.

Gabrielsson, A. (1993). Strong experiences with music. In Juslin, P. N., \& Sloboda, J. (Eds.), Handbook of music and emotion: Theory, research, applications (pp. 547-574). Oxford, UK: Oxford University Press.

Garza-Villarreal, E., Jiang, Z., Vuust, P., Alcauter, S., Vase, L., Pasaye, E., ... Barrios, F. (2015). Music reduces pain and increases resting state fMRI BOLD signal amplitude in the left angular gyrus in fibromyalgia patients. Frontiers in Psychology, 6, 1051. Retrieved from https://doi.org/10.1371/ journal.pone.0013812

Garza-Villarreal, E. A., Wilson, A. D., Vase, L., Brattico, E., Barrios, F. A., Jensen, T. S., ... Vuust, P. (2014). Music reduces pain and increases functional mobility in fibromyalgia. Frontiers in Psychology, 5. DOI: $10.3389 /$ fpsyg.2014.00090

Gingras, B., Honing, H., Peretz, I., Trainor, L. J., \& Fisher, S. E. (2015). Defining the biological bases of individual differences in musicality. Philosophical Transactions of the Royal Society of London. Series B, Biological Sciences, 370(1664), 20140092.

Goldstein, A. (1980). Thrills in response to music and other stimuli. Physiological Psychology, 8(1), $126-$ 129.

Grewe, O., Nagel, F., Kopiez, R., \& Altenmüller, E. (2005). How does music arouse 'chills'? Investigating strong emotions, combining psychological, physiological, and psychoacoustical methods. Annals of the New York Academy of Sciences, 1060, 446-449.

Grewe, O., Nagel, F., Kopiez, R., \& Altenmüller, E. (2007). Listening to music as a re-creative process: Physiological, psychological, and psychoacoustical correlates of chills and strong emotions. Music Perception: An Interdisciplinary Journal, 24(3), 297-314.

Habibi, A., Cahn, B. R., Damasio, A., \& Damasio, H. (2016). Neural correlates of accelerated auditory processing in children engaged in music training. Developmental Cognitive Neuroscience, 21, 1-14.

Habibi, A., \& Damasio, A. (2014). Music, feelings, and the human brain. Psychomusicology: Music, Mind, and Brain, 24(1), 92-102.

Hargreaves, D. J., \& North, A. C. (2010). Experimental aesthetics and liking for music. In Juslin, P. N., \& Sloboda, J. (Eds.), Handbook of music and emotion: Theory, research, applications (pp. 515-546). Oxford, UK: Oxford University Press.

Harrison, L., \& Loui, P. (2014). Thrills, chills, frissons, and skin orgasms: Toward an integrative model of transcendent psychophysiological experiences in music. Frontiers in Psychology, 5, 790. Retrieved from https://doi.org/10.3389/fpsyg.2014.00790

Howe, M. W., Tierney, P. L., Sandberg, S. G., Phillips, P. E. M., \& Graybiel, A. M. (2013). Prolonged dopamine signalling in striatum signals proximity and value of distant rewards. Nature, (7464), 575-579.

Huron, D. (2011). Why is sad music pleasurable? A possible role for prolactin. Musicae Scientiae: The Journal of the European Society for the Cognitive Sciences of Music, 15(2), 146-158.

Hyde, K. L., Lerch, J., Norton, A., Forgeard, M., Winner, E., Evans, A. C., \& Schlaug, G. (2009). Musical training shapes structural brain development. The Journal of Neuroscience: The Official Journal of the Society for Neuroscience, 29(10), 3019-3025.

Ishizu, T., \& Zeki, S. (2011). Toward a brain-based theory of beauty. PloS One, 6(7), e21852. DOI: 10.1371/journal.pone.0021852

James, C. E., Britz, J., Vuilleumier, P., Hauert, C.-A., \& Michel, C. M. (2008). Early neuronal responses in right limbic structures mediate harmony incongruity processing in musical experts. NeuroImage, 42(4), 1597-1608. 
Järvelä, I. (2018). Genomics studies on musical aptitude, music perception, and practice. Annals of the New York Academy of Sciences, 1423(1), 82-91.

Juslin, P. N. (2013a). From everyday emotions to aesthetic emotions: Towards a unified theory of musical emotions. Physics of Life Reviews, 10(3), 235-266.

Juslin, P. N. (2013b). The value of a uniquely psychological approach to musical aesthetics: Reply to the commentaries on 'A unified theory of musical emotions.' Physics of Life Reviews, 10(3), 281-286.

Juslin, P. N., \& Västfjäll, D. (2008). Emotional responses to music: The need to consider underlying mechanisms. The Behavioral and Brain Sciences, 31(5), 559-575; discussion 575-621.

Koelsch, S. (2010). Towards a neural basis of music-evoked emotions. Trends in Cognitive Sciences, 14(3), 131-137.

Koelsch, S. (2014). Brain correlates of music-evoked emotions. Nature Reviews. Neuroscience, 15(3), 170180.

Kraus, N., \& White-Schwoch, T. (2017). Neurobiology of everyday communication: What have we learned from music? The Neuroscientist: A Review Journal Bringing Neurobiology, Neurology and Psychiatry, 23(3). Retrived from https://doi.org10.1177/1073858416653593

Kreutz, G., Bongard, S., Rohrmann, S., Hodapp, V., \& Grebe, D. (2004). Effects of choir singing or listening on secretory immunoglobulin A, cortisol, and emotional state. Journal of Behavioral Medicine, 27(6), 623-635.

Kringelbach, M. L., \& Berridge, K. C. (2017). The affective core of emotion: Linking pleasure, subjective well-being, and optimal metastability in the brain. Emotion Review: Journal of the International Society for Research on Emotion, 9(3), 191-199.

Kumar, S., Forster, H. M., Bailey, P., \& Griffiths, T. D. (2008). Mapping unpleasantness of sounds to their auditory representation. The Journal of the Acoustical Society of America, 124(6), 3810-3817.

LeDoux, J. E. (2017). Semantics, surplus meaning, and the science of fear. Trends in Cognitive Sciences, 21(5), 303-306.

Liégeois-Chauvel, C., Bénar, C., Krieg, J., Delbé, C., Chauvel, P., Giusiano, B., \& Bigand, E. (2014). How functional coupling between the auditory cortex and the amygdala induces musical emotion: a single case study. Cortex: A Journal Devoted to the Study of the Nervous System and Behavior, 60, 82-93.

Liu, C., Abu-Jamous, B., Brattico, E., \& Nandi, A. K. (2017). Towards tunable consensus clustering for studying functional brain connectivity during affective processing. International Journal of Neural Systems, 27(2), 1650042.

Liu, C., Brattico, E., Abu-Jamous, B., Pereira, C. S., Jacobsen, T., \& Nandi, A. K. (2017). Effect of explicit evaluation on neural connectivity related to listening to unfamiliar music. Frontiers in Human Neuroscience, 11, 611. Retrieved from https://doi.org/10.3389/fnhum.2017.00611

MacDonald, R. A. R., Mitchell, L. A., Dillon, T., Serpell, M. G., Davies, J. B., Ashley, E. A. (2003). An empirical investigation of the anxiolytic and pain reducing effects of music. Psychology of Music, 31(2), $187-203$.

MacDonald, R., Kreutz, G., \& Mitchell, L. (2013). Music, health, and wellbeing. Oxford, UK: Oxford University Press.

Martínez-Molina, N., Mas-Herrero, E., Rodríguez-Fornells, A., Zatorre, R. J., \& Marco-Pallarés, J. (2016). Neural correlates of specific musical anhedonia. Proceedings of the National Academy of Sciences, 113(46), E7337-E7345.

Matrone, C., \& Brattico, E. (2015). The power of music on Alzheimer's disease and the need to understand the underlying molecular mechanisms. Journal of Alzheimer's Disease and Parkinsonism, 05(03). DOI: dx.doi.org10.4172/2161-0460.1000196

Mencke, I., Omigie, D., Wald-Fuhrmann, M., \& Brattico, E. (2018). Atonal music: Can uncertainty lead to pleasure? Frontiers in Neuroscience, 12, 979. DOI: 10.3389/fnins.2018.00979

Miendlarzewska, E. A., \& Trost, W. J. (2013). How musical training affects cognitive development: Rhythm, reward and other modulating variables. Frontiers in Neuroscience, 7, 279. Retrieved from https://doi.org/10.3389/fnins.2013.00279

Mitchell, L. A., \& MacDonald, R. A. R. (2006). An experimental investigation of the effects of preferred and relaxing music listening on pain perception. Journal of Music Therapy, 43(4), 295-316. 
Mitra, A., \& Raichle, M. E. (2018). Principles of cross-network communication in human resting state fMRI. Scandinavian Journal of Psychology, 59(1), 83-90.

Molnar-Szakacs, I., \& Overy, K. (2006). Music and mirror neurons: From motion to 'e'motion. Social Cognitive and Affective Neuroscience 1(3), 235-241.

Montoya, R. M., Horton, R. S., Vevea, J. L., Citkowicz, M., \& Lauber, E. A. (2017). A re-examination of the mere exposure effect: The influence of repeated exposure on recognition, familiarity, and liking. Psychological Bulletin, 143(5), 459-498.

Mori, K., \& Iwanaga, M. (2017). Two types of peak emotional responses to music: The psychophysiology of chills and tears. Scientific Reports, 7, 46063.

Nieminen, S., Istók, E., Brattico, E., Tervaniemi, M., \& Huotilainen, M. (2011). The development of aesthetic responses to music and their underlying neural and psychological mechanisms. Cortex: A Journal Devoted to the Study of the Nervous System and Behavior, 47(9), 1138-1146.

Oikkonen, J., Onkamo, P., Järvelä, I., \& Kanduri, C. (2016). Convergent evidence for the molecular basis of musical traits. Scientific Reports, 6, 39707.

Panksepp, J., \& Bernatzky, G. (2002). Emotional sounds and the brain: The neuro-affective foundations of musical appreciation. Behavioural Processes, 60(2), 133-155.

Parkinson, B., Totterdell, P., Briner, R. B., \& Reynolds, S. (1996). Changing moods: The psychology of mood and mood regulation. London, UK: Longman.

Pauwels, E. K. J., Volterrani, D., Mariani, G., \& Kostkiewics, M. (2014). Mozart, music and medicine: Medical Principles and Practice. International Journal of the Kuwait University, Health Science Centre, 23(5), 403-412.

Peck, K. J., Girard, T. A., Russo, F. A., \& Fiocco, A. J. (2016). Music and memory in Alzheimer's disease and the potential underlying mechanisms. Journal of Alzheimer's Disease: JAD, 51(4), 949-959.

Pelowski, M., Markey, P. S., Forster, M., \& Gerger, G. (2017). Move me, astonish me ... delight my eyes and brain: The Vienna integrated model of top-down and bottom-up processes in Art Perception (VIMAP) and corresponding affective, evaluative, and neurophysiological correlates. Physics of Life Reviews, 21, 80-125.

Peretz, I., Cummings, S., \& Dubé, M.-P. (2007). The genetics of congenital amusia (tone deafness): A family-aggregation study. American Journal of Human Genetics, 81(3), 582-588.

Peretz, I., \& Vuvan, D. T. (2017). Prevalence of congenital amusia. European Journal of Human Genetics: EJHG, 25(5), 625-630.

Petrovsky, D., Cacchione, P. Z., \& George, M. (2015). Review of the effect of music interventions on symptoms of anxiety and depression in older adults with mild dementia. International Psychogeriatrics / IPA, 27(10), 1661-1670.

Plomp, R. (1976). Aspects of tone sensation: A psychophysical study. New York, NY: Academic Press.

Preissler, M. A., \& Bloom, P. (2008). Two-year-olds use artist intention to understand drawings. Cognition, 106(1), 512-518.

Putkinen, V., Tervaniemi, M., Saarikivi, K., Ojala, P., \& Huotilainen, M. (2014). Enhanced development of auditory change detection in musically trained school-aged children: A longitudinal event-related potential study. Developmental Science, 17(2), 282-297.

Quarto, T., Fasano, M. C., Taurisano, P., Fazio, L., Antonucci, L. A., Gelao, B., \& ... Brattico, E. (2017). Interaction between DRD2 variation and sound environment on mood and emotion-related brain activity. Neuroscience, 341, 9-17.

Reybrouck, M., \& Brattico, E. (2015). Neuroplasticity beyond sounds: neural adaptations following longterm musical aesthetic experiences. Brain Sciences, 5(1), 69-91.

Reybrouck, M., Vuust, P., \& Brattico, E. (2018). Brain connectivity networks and the aesthetic experience of music. Brain Sciences, 8(6). DOI: 10.3390/brainsci8060107

Rickard, N. S. (2004). Intense emotional responses to music: A test of the physiological arousal hypothesis. Psychology of Music, 32(4), 371-388.

Rogenmoser, L., Kernbach, J., Schlaug, G., \& Gaser, C. (2018). Keeping brains young with making music. Brain Structure \& Function, 223(1), 297-305. 
Sachs, M. E., Ellis, R. J., Schlaug, G., \& Loui, P. (2016). Brain connectivity reflects human aesthetic responses to music. Social Cognitive and Affective Neuroscience, 11(6), 884-891.

Salimpoor, V. N., Benovoy, M., Larcher, K., Dagher, A., \& Zatorre, R. J. (2011). Anatomically distinct dopamine release during anticipation and experience of peak emotion to music. Nature Neuroscience, 14(2), 257-262.

Salimpoor, V. N., Benovoy, M., Longo, G., Cooperstock, J. R., \& Zatorre, R. J. (2009). The rewarding aspects of music listening are related to degree of emotional arousal. PloS One, 4(10), e7487. Retrieved from https://doi.org/10.1371/journal.pone.0007487

Salimpoor, V. N., van den Bosch, I., Kovacevic, N., McIntosh, A. R., Dagher, A., \& Zatorre, R. J. (2013). Interactions between the nucleus accumbens and auditory cortices predict music reward value. Science, 340(6129), 216-219.

Salimpoor, V. N., Zald, D. H., Zatorre, R. J., Dagher, A., \& McIntosh, A. R. (2015). Predictions and the brain: How musical sounds become rewarding. Trends in Cognitive Sciences, 19(2), 86-91.

Salimpoor, V. N., \& Zatorre, R. J. (2013). Neural interactions that give rise to musical pleasure. Psychology of Aesthetics, Creativity, and the Arts, 7(1), 62-75.

Särkämö, T. (2018). Music for the ageing brain: Cognitive, emotional, social, and neural benefits of musical leisure activities in stroke and dementia. Dementia, 17(6), 670-685.

Särkämö, T., Ripollés, P., Vepsäläinen, H., Autti, T., Silvennoinen, H. M., Salli, E., ... Rodríguez-Fornells, A. (2014). Structural changes induced by daily music listening in the recovering brain after middle cerebral artery stroke: A voxel-based morphometry study. Frontiers in Human Neuroscience, 8, 245. Retrieved from https://doi.org/10.3389/fnhum.2014.00245

Särkämö, T., \& Sihvonen, A. J. (2018). Golden oldies and silver brains: Deficits, preservation, learning, and rehabilitation effects of music in ageing-related neurological disorders. Cortex: A Journal Devoted to the Study of the Nervous System and Behavior, 109, 104-123.

Särkämö, T., Tervaniemi, M., Laitinen, S., Numminen, A., Kurki, M., Johnson, J. K., \& Rantanen, P. (2014). Cognitive, emotional, and social benefits of regular musical activities in early dementia: Randomized controlled study. The Gerontologist, 54(4), 634-650.

Scherer, K., \& Zentner, M. (2008). Music evoked emotions are different - more often aesthetic than utilitarian. The Behavioral and Brain Sciences, 31(05).

Schindler, I., Hosoya, G., Menninghaus, W., Beermann, U., Wagner, V., Eid, M., \& Scherer, K. R. (2017). Measuring aesthetic emotions: A review of the literature and a new assessment tool. PloS One, 12(6), e0178899. Retrieved from https://doi.org/10.1371/journal.pone.0178899

Schlaug, G. (2003). The brain of musicians. In Zatorre, R., \& Peretz, I. (Eds.), The cognitive neuroscience of music (pp. 366-381). Oxford, UK: Oxford University Press.

Schubert, E., \& North, A. C. (2016). Aesthetic experience explained by the affect-space framework. Empirical Musicology Review: EMR, 11(3-4), 330-345.

Seashore, C. E. (1911). Questionnaire for Seashore Pitch Discrimination Test. PsycTESTS Dataset.

Singer, W., Panford-Walsh, R., \& Knipper, M. (2014). The function of BDNF in the adult auditory system. Neuropharmacology, 76 Pt C, 719-728.

Sloboda, J. A. (1991). Music structure and emotional response: Some empirical findings. Psychology of Music, 19(2), 110-120.

Snapper, L., Oranç, C., Hawley-Dolan, A., Nissel, J., \& Winner, E. (2015). Your kid could not have done that: Even untutored observers can discern intentionality and structure in abstract expressionist art. Cognition, 137, 154-165.

Stark, E. A., Vuust, P., \& Kringelbach, M. L. (2018). Music, dance, and other art forms: New insights into the links between hedonia (pleasure) and eudaimonia (well-being). Progress in Brain Research, 237, $129-152$.

Steinbeis, N., \& Koelsch, S. (2009). Understanding the intentions behind man-made products elicits neural activity in areas dedicated to mental state attribution. Cerebral Cortex, 19(3), 619-623.

Sumpf, M., Jentschke, S., \& Koelsch, S. (2015). Effects of aesthetic chills on a cardiac signature of emotionality. PloS One, 10(6), e0130117. Retrieved from https://doi.org/10.1371/journal.pone.0130117 
Taruffi, L., \& Koelsch, S. (2014). The paradox of music-evoked sadness: An online survey. PloS One, 9(10), e110490. Retrieved from https://doi.org/10.1371/journal.pone.0110490

Taruffi, L., Pehrs, C., Skouras, S., \& Koelsch, S. (2017). Effects of sad and happy music on mind-wandering and the default mode network. Scientific Reports, 7(1), 14396.

Terhardt, E. (1974). Pitch, consonance, and harmony. The Journal of the Acoustical Society of America, 55(5), 1061-1069.

Thaut, M. H. (2015). Music as therapy in early history. Progress in Brain Research, 217, 143-158.

Thaut, M. H., \& Wheeler, B. L. (1993). Music therapy. In Juslin, P. N., \& Sloboda, J. (Eds.), Handbook of music and emotion: Theory, research, applications (pp. 818-848). Oxford, UK: Oxford University Press.

Tiihonen, M., Brattico, E., Maksimainen, J., Wikgren, J., \& Saarikallio, S. (2017). Constituents of music and visual-art related pleasure: A critical integrative literature review. Frontiers in Psychology, 8 , 1218. Retrieved from https://doi.org/10.3389/fpsyg.2017.01218

Trehub, S. E. (2003). Musical predispositions in infancy: An update. In The cognitive neuroscience of music. Oxford, UK: Oxford University Press.

Ukkola-Vuoti, L., Kanduri, C., Oikkonen, J., Buck, G., Blancher, C., Raijas, P., ... Järvelä, I. (2013). Genome-wide copy number variation analysis in extended families and unrelated individuals characterized for musical aptitude and creativity in music. PloS One, 8(2), e56356. Retrived from https:// doi.org/10.1371/journal.pone.0056356

Vuoskoski, J. K., \& Eerola, T. (2017). The pleasure evoked by sad music is mediated by feelings of being moved. Frontiers in Psychology, 8, 439. Retrieved from https://doi.org/10.3389/fpsyg.2017.00439

Wilkins, R. W., Hodges, D. A., Laurienti, P. J., Steen, M., \& Burdette, J. H. (2014). Network science and the effects of music preference on functional brain connectivity: From Beethoven to Eminem. Scientific Reports, 4, 6130.

Y. Wan, C., \& Schlaug, G. (2013). Brain plasticity induced by musical training. In Deutsch, D. (Ed.), The psychology of music (Third Edition) (pp. 565-581). San Diego, USA: Academic Press.

Zajonc, R. B. (1968). Attitudinal effects of mere exposure. Journal of Personality and Social Psychology, 9(2, Pt.2), 1-27.

Zatorre, R. J., \& Salimpoor, V. N. (2013). From perception to pleasure: Music and its neural substrates. Proceedings of the National Academy of Sciences of the United States of America, 110 (Suppl 2), 1043010437. 